\title{
Regional Cortical Thickness and Subcortical Volume Changes Are Associated with Cognitive Impairments in the Drug-Naive Patients with Late-Onset Depression
}

\author{
Hyun Kook Lim', Won Sang Jung ${ }^{2}$, Kook Jin Ahn ${ }^{3}$, Wang Youn Won ${ }^{4}$, Changtae Hahn ${ }^{5}$, Seung Yup Lee', \\ InSeong Kim ${ }^{6}$ and Chang Uk Lee*,5 \\ 'Department of Psychiatry, Saint Vincent Hospital, The Catholic University of Korea, Suwon, Korea; ${ }^{2}$ Department of Radiology, Saint Vincent \\ Hospital, The Catholic University of Korea, Suwon, Korea; ${ }^{3}$ Department of Radiology, Seoul Saint Mary's Hospital, The Catholic University of \\ Korea, Seoul, Korea; ${ }^{4}$ Department of Psychiatry, Saint Paul Hospital, The Catholic University of Korea, Seoul, Korea; ${ }^{5}$ Department of Psychiatry, \\ Seoul Saint Mary's Hospital, The Catholic University of Korea, Seoul, Korea; ${ }^{6}$ SIEMENS Healthcare, Seoul, Korea
}

Previous studies have shown an association between late-onset depression (LOD) and cognitive impairment in older adults. However, the neural correlates of this relationship are not yet clear. The aim of this study was to investigate the differences in both cortical thickness and subcortical volumes between drug-naive LOD patients and healthy controls and explore the relationship between LOD and cognitive impairments. A total of 48 elderly, drug-naive patients with LOD and 47 group-matched healthy control subjects underwent $3 T$ MRI scanning, and the cortical thickness was compared between the groups in multiple locations, across the continuous cortical surface. The subcortical volumes were also compared on a structure-by-structure basis. Subjects with LOD exhibited significantly decreased cortical thickness in the rostral anterior cingulate cortex, the medial orbitofrontal cortex, dorsolateral prefrontal cortex, the superior and middle temporal cortex, and the posterior cingulate cortex when compared with healthy subjects (all $p<0.05$, false discovery rate corrected). Reduced volumes of the right hippocampus was also observed in LOD patients when compared with healthy controls $(p<0.00 \mathrm{I})$. There were significant correlations between memory functions and cortical thickness of medial temporal, isthmus cingulate, and precuneus $(p<0.00 \mathrm{I})$. This study was the first study to explore the relationships between the cortical thickness/subcortical volumes and cognitive impairments of drug-naive patients with LOD. These structural changes might explain the neurobiological mechanism of LOD as a risk factor of dementia.

Neuropsychopharmacology (2012) 37, 838-849; doi:I0.1038/npp.20 I 1.264; published online 2 November 201 I

Keywords: late-onset depression; cortical thickness; subcortical volumes

\section{INTRODUCTION}

Depression can be a significant health-care risk for older adults and a major cause of disability (Crocco et al, 2010).

In a subset of elderly depression, late-onset depression (LOD), of which the first episode occurs later in life, exhibits certain unique clinical features (Alexopoulos et al, 1988; Krishnan et al, 1995, 1997; Schweitzer et al, 2002); thus, the LOD patients are less likely to have psychiatric comorbidity or a family history of depression when compared with early-onset depression subgroups (Brown

*Correspondence: Dr CU Lee, Department of Psychiatry, Seoul Saint Mary's Hospital, The Catholic University of Korea, 505 Banpo dong Seochogu, Seoul, I37-50 Republic of Korea, Tel: + 8222258 6082, Fax: + 822594 3870, E-mail: jihan@catholic.ac.kr

Received 4 May 2011; revised 6 September 2011; accepted 23 September 2011 et al, 1984; Krishnan et al, 1997). On the other hand, the LOD patients are more likely to have associated medical comorbidity (Alexopoulos et al, 1997; Emery and Oxman, 1992; Krishnan, 2002; Sheline, 2003), greater cognitive deficits, and increased risk of developing dementia (Salloway et al, 1996; Schweitzer et al, 2002). Furthermore, individuals with amnestic mild cognitive impairment (MCI) with depressive symptoms show increased propensity for Alzheimer's disease (AD), especially when they are also depressed (Apostolova and Cummings, 2008; Modrego and Ferrndez, 2004). In this regard, there might be an association between LOD and dementia, as previously shown in the literature (Schweitzer et al, 2002). However, the neural correlates of this relationship are still poorly understood.

To date, several morphometric analyses measured the gray matter volume or the density difference in the LOD using manually delineating region-of-interest (ROI) methods, 
voxel-based morphometry (VBM), and the cortical patternmatching method (Ballmaier et al, 2004a, 2008; Egger et al, 2008; Hwang et al, 2010; Janssen et al, 2007; Ries et al, 2009). Although they suggested that patients with LOD showed gray matter volume reductions in the frontal, temporal, and parietal regions and in the subcortical structures when compared with the healthy controls, the results were sometimes rather inconsistent. These might be attributable to the differences in the medication status, comorbid vascular diseases, small sample size, analysis methods, and illness chronicity. Moreover, most of the studies did not use the comprehensive neuropsychological battery of test; therefore, it has been hard to investigate the precise relationship between LOD and cognitive impairments.

In addition to the previous whole-brain voxel-wise analysis, other automated techniques have been developed to estimate the cortical thickness in MRI scans (Fischl and Dale, 2000; Lerch et al, 2005). These methods aim to identify the difference in width of the cortical gray matter on the surface of the brain, without a priori consumption. A previous study suggested that the cortical thickness measurement can be used to selectively investigate atrophy, whereas VBM provides a mixed measure of the cortical gray matter, including the cortical surface area or cortical folding, as well as the cortical thickness (Hutton et al, 2009).

To our best knowledge, there have been no studies to explore cortical thickness change in the LOD. Although only one study has tried to investigate the cortical thickness of elderly depression with early onset, the results showed no difference of cortical thickness between patients with latelife depression and the controls (Koolschijn et al, 2010). It has been suggested that this might be attributable to the relatively small sample size ( $n=28$ of elderly depression), moderate depressive symptoms, only female subjects, lack of information on the subjects, and medication effects. Hence, we tried to explore the cortical thickness and subcortical volumes of drug-naive LOD patients in a relatively larger sample to overcome the aforementioned problems in the cortical thickness measurement of depression. In addition, we tried to investigate the relationships between the cortical thickness and cognitive impairments of the LOD using a comprehensive neuropsychological battery. Throughout these, we sought the distinctive neural correlates of the LOD with cognitive impairments and their clinical implications. Several previous neuroimaging studies have also suggested the structural abnormalities in the fronto-striatal-limbic area of the patients with depressive disorders (Koolschijn et al, 2009; Savitz and Drevets, 2009). Therefore, we hypothesized that both the cortical thickness and the subcortical volumes of fronto-striatal-limbic structures might be reduced and show distinctive correlation patterns with the neuropsychological performances in LOD compared with healthy controls.

\section{SUBJECTS AND METHODS}

\section{Subjects}

In this study, 48 patients with a lifetime diagnosis of a major depressive disorder were included. They were recruited through the outpatient psychogeriatric clinic of St Vincent
Hospital located at Suwon, South Korea, from October 2009 to October 2010.

The inclusion criteria of the patient group were as follows: (1) patients aged > 60 years; (2) DSM-IV TR diagnosis of major depressive disorder established with the Mini-International Neuropsychiatric Interview (MINI) (Sheehan et al, 1998); (3) the first episode of major depression after the age of 60 years; (4) total score of $>10$ on the 17-item Hamilton Depression Rating Scale (HAM-D ${ }_{17}$ ) (Hamilton, 1967); (5) the Korean version of the Mini-Mental State Examination (MMSE) score of $>26$ (Park and Kwon, 1990); and (6) global Clinical Dementia Rating score of 0 (Morris, 1993).

The exclusion criteria for the patient group were as follows: (1) patients with a presumptive diagnosis of dementia and other neurological or medical conditions that diminish the cognitive function (eg, hypothyroidism); (2) a history of other psychiatric disorders (eg, schizophrenia, delusional disorder, substance abuse); (3) unstable medical conditions (eg, poorly controlled hypertension, angina, or diabetes); (4) any clinically relevant abnormal electrocardiograms or laboratory findings or brain MRI findings (eg, serious deep white matter hyper intensities, lacuna infarction, or brain tumors), and (5) patients taking any psychotropic medications (eg, antidepressant, benzodiazepines, and antipsychotics).

Subjects were screened with a self-report health questionnaire that reviewed both the demographic data and the medical history. The depression duration was assessed in an interview by using the life-chart methodology. The depression severity at the time of the scan was measured with the HAM- $\mathrm{D}_{17}$. The cognitive functions of all the subjects were assessed with the Korean version of Consortium to Establish a Registry for Alzheimer's Disease (CERAD-K), including Verbal Fluency (VF), 15-item Boston Naming Test (BNT), MMSE, Word List Memory (WLM), Word List Recall (WLR), Word List Recognition (WLRc), Constructional Praxis (CP), and Constructional Recall (CR) (Lee et al, 2002). In addition, the Stroop Word-Color Interference test was administered for the measurement of executive functioning (Stroop, 1935). All clinical measurements were carried out on the day of the MRI scanning.

Through advertisement in the local newspaper, 47 healthy control participants were recruited within the community. Control subjects were matched to the patients on age, handedness, and level of education. Furthermore, control subjects were given the same self-report health questionnaire as the patients, thus enabling matching on health status. Exclusion criteria were similar to the patient group, with the addition of excluding those with any current or past Axis I psychiatric diagnosis, as established by the MINI and medication use. A clinical neuroradiologist (WSJ) examined the brain MRIs of all the subjects; no gross abnormalities were reported in any participant and showed normal-appearing white matter (Huang et al, 2007). All subjects were right handed and nonsmokers with no history of smoking. None of the subjects had any life-time history of taking any psychotropic medication, which was verified during an interview with each patient and members of his/her family. In addition, no subjects had any history of psychiatric or neurologic treatment, which was verified by thorough review of the medical records 
of the subjects. The psychometric evaluations and the clinical diagnosis were performed by two board-certified psychiatrists (HKL and CUL).

The study was conducted in accordance with the ethical and safety guidelines set forth by the institutional review board of the Catholic University of Korea. Written consent was obtained from all subjects participating in the study.

\section{MRI Acquisition and Preprocessing}

All participants underwent MRI scans on a 3-Tesla whole body scanner equipped with an 8-channel phased-array head coil (Verio, Siemens, Erlangen, Germany). The scanning parameters of the T1-weighted three-dimensional magnetization-prepared rapid gradient-echo (3D-MPRAGE) sequences were as follows: $\mathrm{TE}=2.5 \mathrm{~ms}$; $\mathrm{TR}=1900 \mathrm{~ms}$; inversion time $(\mathrm{TI})=900 \mathrm{~ms}$; flip angle $(\mathrm{FA})=9^{\circ}$; $\mathrm{FOV}=$ $250 \times 250 \mathrm{~mm} ; \quad$ matrix $=256 \times 256$; voxel size $=1.0 \times$ $1.0 \times 1.0 \mathrm{~mm}^{3}$.

For cortical reconstruction and volumetric segmentation of the whole brain, Freesurfer image analysis suite (version 5.0, http://surfer.nmr.mgh.harvard.edu), which is documented and freely available online, was used. The technical details of these procedures have been described in previous publications (Dale et al, 1999; Fischl and Dale, 2000). Briefly, the processing stream includes a Talairach transform of each subject's native brain, removal of the nonbrain tissue, and segmentation of the gray matter/white matter (GM/WM) tissue. The cortical surface of each hemisphere was inflated to an average spherical surface to locate both the pial surface and the GM/WM boundary. The entire cortex of each subject was visually inspected, and any topological defects were corrected manually, blind to the subject's identity. The cortical thickness was computed as the shortest distance between the pial surface and the GM/WM boundary at each point across the cortical mantle. The global mean cortical thickness for each subject was computed by averaging the cortical thickness at each vertex, right and left hemispheres separately, and was used in the statistical analyses. The regional thickness value at each vertex for each subject was mapped to the surface of an average brain template allowing visualization of data across the entire cortical surface (described at http://surfer. nmr.mgh.harvard.edu/fswiki/FsAverage). In addition, the entire cerebral cortex was parcellated into 34 regions (Desikan et al, 2006; Fischl et al, 2004), and a variety of surface-based data, including maps of cortical volume and surface area as well as curvature and sulcal depth, were created. Data were resampled for all subjects onto a common spherical coordinate system (Fischl et al, 1999). The cortical map of each subject was smoothed with a Gaussian kernel of 10-mm full width at half-maximum for the entire cortex analyses. The subcortical volumes were obtained from the automated procedure for volumetric measures of the brain structures implemented in Freesurfer. In all, 27 volumetric measures were investigated and extracted seven subcortical structures (white matter, caudate, thalamus, pallidum, putamen, hippocampus, and amygdala) from each hemisphere. The reliability studies on the measuring cortical thickness and subcortical volumes reported that within-scanner variabilities of cortical thickness and subcortical volume measurements using Freesurfer were estimated to be $<0.03 \mathrm{~mm}$ and $4.3 \%$, respectively (Han et al, 2006; Jovicich et al, 2009).

\section{Statistical Analyses}

Statistical analyses for demographic data (Table 1) were performed with the Statistical Package for Social Sciences software (SPSS, version 12.0, Chicago, IL). Assumptions for normality were tested for all continuous variables. Normality was tested using the Kolmogorov-Smirnov test. All variables were normally distributed. The independent $t$-test and the $\chi^{2}$ test were used to assess potential differences between the LOD groups and healthy control groups for all demographic variables. All statistical analyses had a two-tailed $\alpha$ level of $<0.05$ for defining statistical significance.

The general linear model (GLM) was implemented at each vertex in the whole brain to identify the brain regions in which LOD patients showed significant differences in cortical thickness relative to controls, using the FreeSurfer's mri_glmfit (described at http://surfer.nmr.mgh.harvard. edu/fswiki/mri_glmfit). The correlation analyses between regional cortical thickness/subcortical volumes and several clinical outcome measures including the HAM- $D_{17}$ scores, CERAD-K scores, Stroop Word-Color Interference test scores, and duration of illness were also conducted. In particular, vertex-wise GLM analyses were performed in the control and LOD groups independently to explore the brain regions that showed significant correlations between cortical thickness and several clinical outcome measures in both controls and LOD patients, using mri_glmfit. The interactions between diagnosis and several clinical outcomes were also examined to compare the differences in regression slopes of the clinical outcome measures between both groups. The effects of age, education, total intracranial volume (TIV), and gender were regressed out in these models. For further investigation of the effect of age on cortical thickness, we conducted regression analysis between the cortical thickness of entire cortex and age in all subjects, the LOD group and the control group, respectively. In addition, we also compared the regression patterns of age and cortical thickness between the LOD group and the control group.

All analyses were performed for the right and left hemispheres separately. The threshold was set at $p<0.05$ (false discovery rate (FDR)) to resolve the problem of multiple comparisons (Genovese et al, 2002).

The seven subcortical structure volumes (ie, total white matter volumes, thalamus, caudate nucleus, putamen, pallidum, hippocampus, and amygdala) were imported into the SPSS 12.0 software for statistical analyses. To assess the main effects of diagnosis (LOD vs control) on the volume of subcortical structures, we used analysis of covariance (ANCOVA) with TIV, education, gender, and age as nuisance variables. In addition, regression analyses were performed to determine the contribution of clinical variables (HAM- $\mathrm{D}_{17}$ scores, CERAD-K scores, Stroop WordColor Interference test scores, and duration of illness) to subcortical structural volumes. For further investigation of the effect of age on subcortical volumes, we conducted the regression analysis between the subcortical volumes and age in all subjects, the LOD group and the control group, 
Table I Demographic and Clinical Characteristics of Study Participants

\begin{tabular}{|c|c|c|c|}
\hline & $\begin{array}{c}\text { Control } \\
\text { group } \\
(N=47)\end{array}$ & $\begin{array}{c}\text { LOD } \\
\text { group } \\
(N=48)\end{array}$ & $P$-value \\
\hline Age (years $\pm S D$ ) & $70.7 \pm 4.37$ & $71.8 \pm 4.8$ & NS \\
\hline Education (years $\pm S D$ ) & $9.2 \pm 4.8$ & $9.3 \pm 4.2$ & NS \\
\hline $\operatorname{Sex}(M / F)$ & $9 / 38$ & $12 / 36$ & NS \\
\hline Duration of illness (years $\pm S D$ ) & 0 & $1.3 \pm 0.5$ & $<0.000$ । \\
\hline HAM-D 17 total score (SD) & $1.9 \pm 1.9$ & $18.9 \pm 6.5$ & $<0.0001$ \\
\hline \multicolumn{4}{|l|}{ CERAD-K battery (SD) } \\
\hline VF & $13.3 \pm 2.9$ & $12.3 \pm 3.7$ & NS \\
\hline BNT & $11.3 \pm 2.1$ & $9.3 \pm 2.4$ & $<0.0001$ \\
\hline MMSE & $28.1 \pm 11$ & $27.1 \pm 1.0$ & 0.001 \\
\hline WLM & $18.2 \pm 3.5$ & $14.6 \pm 4.0$ & $<0.0001$ \\
\hline $\mathrm{CP}$ & $9.4 \pm 1.5$ & $8.1 \pm 1.9$ & NS \\
\hline WLR & $6.4 \pm 1.9$ & $4.3 \pm 1.8$ & $<0.0001$ \\
\hline WLRc & $9.3 \pm 1.0$ & $8.1 \pm 1.9$ & $<0.000$ । \\
\hline$C R$ & $6.7 \pm 2.9$ & $4.8 \pm 3.0$ & 0.003 \\
\hline Stroop test reaction time (seconds $\pm S D$ ) & $30.2 \pm 5.7$ & $42.1 \pm 16.8$ & 0.003 \\
\hline Stroop test error (SD) & $1.5 \pm 1.4$ & $2.5 \pm 2.6$ & NS \\
\hline Hypertension ${ }^{\mathrm{a}}, n(\%)$ & $5(10.6)$ & $6(12.5)$ & NS \\
\hline Diabetes $^{b}, n(\%)$ & $3(6.4)$ & $2(4.1)$ & NS \\
\hline Dyslipidemiac, n (\%) & $2(4.1)$ & I $(2.1)$ & NS \\
\hline Stroke or TIA, n (\%) & 0 & 0 & NS \\
\hline History of heart disease, $n$ (\%) & 0 & 0 & NS \\
\hline Current smokers, $n(\%)$ & 0 & 0 & NS \\
\hline History of smoking, n (\%) & 0 & 0 & NS \\
\hline Antihypertensive agents, $n$ (\%) & $5(10.6)$ & $6(12.5)$ & NS \\
\hline Hypoglycemic agents, n (\%) & $3(6.4)$ & I (2.1) & NS \\
\hline Lipid-lowering agents, n (\%) & $2(4.1)$ & I (2.1) & NS \\
\hline Body mass index $\left(\mathrm{kg} / \mathrm{m}^{2} \pm \mathrm{SD}\right)$ & $24.2 \pm 3.9$ & $23.7 \pm 4.1$ & NS \\
\hline Systolic blood pressure $(\mathrm{mm} \mathrm{Hg} \pm \mathrm{SD})$ & $128.2 \pm 7.3$ & $127.5 \pm 8.1$ & NS \\
\hline Diastolic blood pressure $(\mathrm{mm} \mathrm{Hg} \pm \mathrm{SD})$ & $83.4 \pm 8.7$ & $82.3 \pm 7.4$ & NS \\
\hline Total cholesterol (mg/dl $\pm \mathrm{SD})$ & $170.8 \pm 23.4$ & $169.9 \pm 25.1$ & NS \\
\hline High-density lipoprotein (mg/dl $\pm S D$ ) & $58.5 \pm 13.2$ & $59.7 \pm 12.5$ & NS \\
\hline Triglycerides (mg/dl $\pm \mathrm{SD})$ & $103.2 \pm 14.9$ & $104.3 \pm 13.7$ & NS \\
\hline Glucose $(\mathrm{mg} / \mathrm{dl} \pm \mathrm{SD})$ & $98.9 \pm 5.7$ & $97.3 \pm 7.4$ & NS \\
\hline
\end{tabular}

Abbreviations: LOD, late-onset depression; NS, statistically not significant; HAM-D 17, 17-item Hamilton Depression Rating Scale; CERAD-K, the Korean version of Consortium to Establish a Registry for Alzheimer's Disease; VF, Verbal Fluency; BNT, I5-item Boston Naming Test; MMSE, Mini Mental Status Examination; WLM, Word List Memory; CP, Constructional Praxis; WLR, Word List Recall; WLRc, Word List Recognition; CR, Constructional Recall; TIA, transient ischemic attack.

aHypertension defined according to the Seventh Report of the Joint National Committee on Prevention, Detection, Evaluation, and Treatment of High Blood Pressure (INC 7) (Chobanian et al, 2003)

${ }^{b}$ Diabetes mellitus defined according to International Diabetes Federation Epidemiology Task Force Consensus Group (Alberti et al, 2005).

'Dyslipidemia defined according to Revised The National Cholesterol Education Program Adult Treatment Panel III criteria (NCEP ATP III) (Grundy et al, 2005.

respectively. In addition, we also compared the regression patterns of age and subcortical volumes between the LOD group and the control group. An uncorrected $p<0.001$ (two tailed) was considered a significant threshold in the statistical difference maps. This threshold, when an a priori hypothesis was present, was approximately equivalent to $p<0.05$ corrected for multiple comparisons (Ashburner et al, 2003; Lyoo et al, 2006).

\section{RESULTS}

\section{Baseline Demographic Data}

Table 1 shows the baseline demographic data in our different subject groups. There was no significant difference in sex, age, and education between the LOD group and the healthy control group. In addition, the degree and frequencies of the vascular risk factors such as hypertension, dyslipidemia, diabetes, obesity, and stroke were not significantly different between the two groups. However, the number of female subjects was significantly larger than the male subjects in each group $(p<0.001)$. Patients with LOD showed significantly poorer performance on BNT, MMSE, WLM, WLR, WLRc, and CR on CERAD-K neuropsychological test, and Stroop Word-Color Interference test $(p<0.05)$.

\section{Cortical Thickness Analysis}

Cortical thickness difference between LOD and control. For the global mean cortical thickness, the LOD group $(n=48)$ showed a significant reduction in both hemispheres when compared with healthy controls $(n=47$; Table 2$)$. A group comparison analysis of the regional cortical thickness between the LOD and the control group showed a significant reduction in the cortical thickness of the LOD group in the left medial orbitofrontal, the dorsolateral prefrontal (DLPFC), pars triangularis, rostral anterior cingulate, superior temporal, middle temporal, precentral, postcentral, lingual, superior parietal, paracentral gyrus and right postcentral, DLPFC, pars opercularis, rostral middle frontal, precuneus, and isthmus cingulate as compared with the control group $(p<0.05$ FDR corrected, Figure 1 and Table 3$)$. No significant cortical thickness reduction was observed in the control group when compared with the LOD group. In addition, there have been no regional cortical thickness differences between male and female subjects in each group.

Correlation analyses between the cortical thickness and clinical outcomes. In the correlation analysis of the cortical thickness of the LOD group with the HAM-D ${ }_{17}$ total scores, we found a significant negative correlation with the left rostral anterior cingulate cortex $(p<0.05$ FDR corrected, Figure 2). There were no significant correlations between the duration of the illness and the cortical thickness of the LOD group. The WLM score in the LOD group revealed a significant positive correlation with the cortical thickness of the left superior temporal, precuneus, and insula and the right precuneus, inferior temporal, and insula $(p<0.05 \mathrm{FDR}$ corrected and Supplementary Table S1). The WLR score in the LOD group revealed a significant positive correlation with the left fusiform, entorhinal, insula, precuneus, precentral and the right isthmus cingulate, insula, supramarginal, inferior parietal, and precuneus $(p<0.05$ FDR corrected, Figure 2 and Supplementary Table S1). The 
Stroop Word-Color Interference test score of the LOD group also displayed a significant negative correlation with the right DLPFC (pars opercularis), superior frontal, precentral, precuneus, medial orbitofrontal, rostral anterior cingulate, rostral middle frontal area, and the left insula $(p<0.05$ FDR corrected, Figure 2 and Supplementary Table $S 1)$. There have been no significant correlations between the VF, BNT, MMSE, CP, WLRc, and CR scores and the cortical thickness of the LOD group under the FDR $<0.05$ conditions. On the other hand, no significant correlations were observed between the neuropsychological test scores and HAM- $\mathrm{D}_{17}$ total scores and cortical thickness in the control group. Age was not correlated significantly with the cortical thickness or cognitive functions in all subjects, the LOD group and the control group, respectively $(p<0.05$ FDR corrected). Furthermore, age-cortical thickness regression patterns were not significantly different between

Table 2 Statistics of Mean Cortical Thickness within Each Hemisphere

\begin{tabular}{|c|c|c|c|c|c|c|}
\hline & \multicolumn{4}{|c|}{ Cortical thickness $(\mathrm{mm})$} & & \\
\hline & \multicolumn{2}{|c|}{$\begin{array}{l}\text { Control group } \\
\quad(N=47)\end{array}$} & \multicolumn{2}{|c|}{$\begin{array}{l}\text { LOD group } \\
(N=48)\end{array}$} & \multicolumn{2}{|c|}{ Statistics } \\
\hline & Mean & SD & Mean & SD & $t$ & $p$ \\
\hline Right hemisphere & 2.50 & 0.10 & 2.40 & 0.11 & 3.44 & 0.001 \\
\hline Left hemisphere & 2.48 & 0.10 & 2.38 & 0.11 & 3.52 & 0.001 \\
\hline
\end{tabular}

Abbreviation: LOD, late-onset depression.

Uncorrected $p<0.00$ I was considered as significant. the LOD group and the control group $(p<0.05$ FDR corrected).

\section{Subcortical Volume Analyses}

For the mean volumes of the total white matter of both hemispheres, no significant difference between the LOD group and the control group was observed (Table 4). There were significant volume reductions in the right hippocampus in the LOD group as compared with the control group $(p=0.001$, Table 4$)$. In addition, there have been no total intracranial volume and subcortical volume differences between male and female subjects in each group. In the correlation analysis, the VF score of the LOD group was significantly correlated with the right putamen $(r=0.513$, $p=0.001$ ) and the hippocampus (Figure 3, $r=0.558$, $p<0.0001)$. We could not find any significant correlations between the score of duration of the illness, HAM- $D_{17}$ BNT, MMSE, WLM, WLR, WLRc, CP, CR, and Stroop Word-Color Interference test score, and subcortical structural volumes in the LOD group. In addition, no significant correlations were observed between the neuropsychological test scores and HAM- $\mathrm{D}_{17}$ total scores and subcortical volumes in the control group. Age was not correlated significantly with the subcortical volumes in all subjects, the LOD group and the control group, respectively. Furthermore, age-subcortical volumes regression patterns were not significantly different between the LOD group and the control group.

\section{DISCUSSION}

To our best knowledge, this is the first study to explore the cortical thinning pattern and the subcortical volume

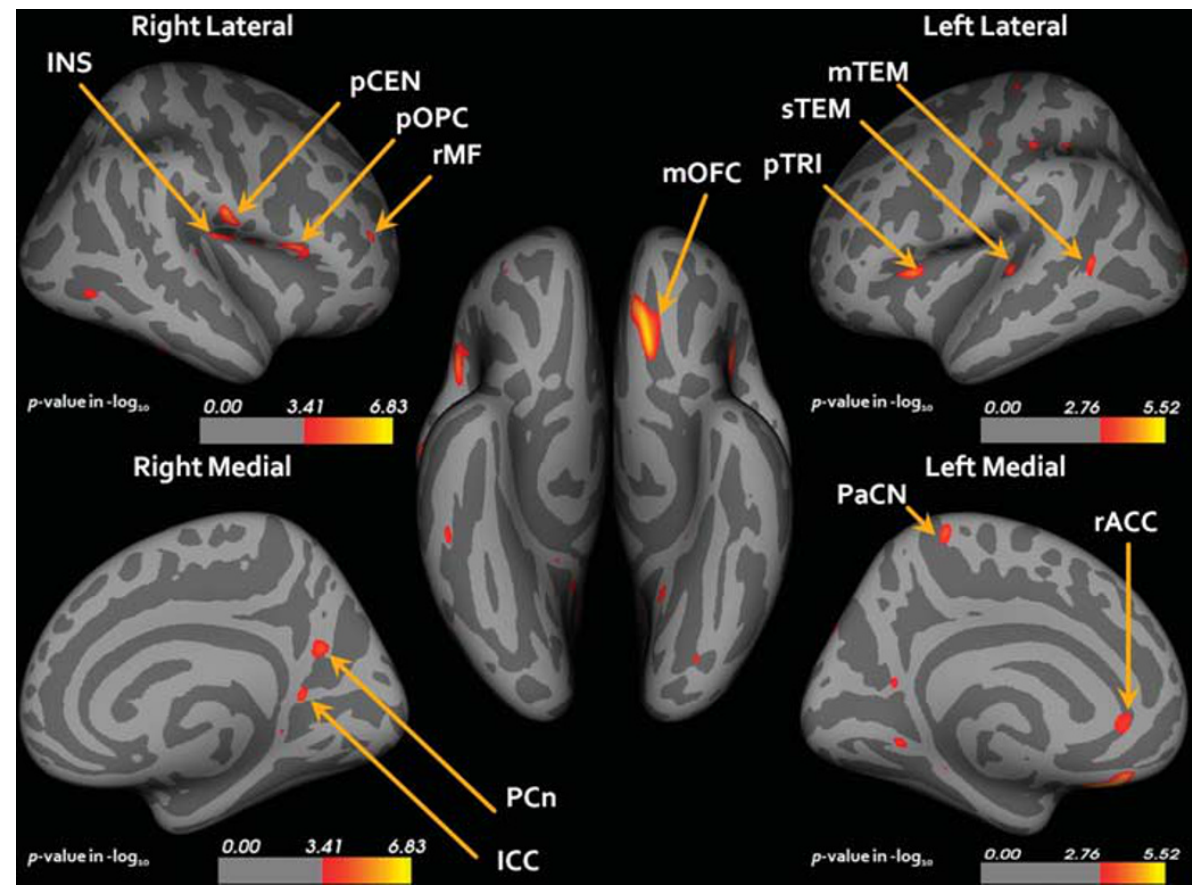

Figure I Statistical maps corrected for age, education, and gender showing reduced cortical thickness in patients with LOD relative to controls ( $p<0.05$ FDR corrected). LOD, late-onset depression; FDR, false discovery rate; INS, insula; $\mathrm{PCEN}$, postcentral; pOPC, pars opercularis; rMF, rostral middle frontal; mOFC, medial orbitofrontal; pTRI, pars triangularis; sTEM, superior temporal; mTEM, middle temporal; PCn, precuneus; ICC, isthmus cingulate; rACC, rostral anterior cingulate. Anatomical terms are used according to the Desikan template (Desikan et al, 2006). 
Table 3 Mean Cortical Thickness for Clusters Where a Significant Cortical Thinning Was Observed in LOD Patients Relative to Healthy Controls (FDR Corrected, $p<0.05$ )

\begin{tabular}{|c|c|c|c|c|c|c|}
\hline \multirow[t]{2}{*}{ Region } & \multirow{2}{*}{$\begin{array}{l}\text { No. of vertexes } \\
\text { in cluster }\end{array}$} & \multirow{2}{*}{$\begin{array}{l}\text { Cluster size, } \\
\left(\mathrm{mm}^{2}\right)\end{array}$} & \multicolumn{2}{|c|}{ Cortical thickness (mm, SD) } & \multirow{2}{*}{$\begin{array}{l}\text { Effect size } \\
\text { (Cohen's d) }\end{array}$} & \multirow[t]{2}{*}{$p$} \\
\hline & & & $\begin{array}{l}\text { Control group } \\
\quad(N=47)\end{array}$ & $\begin{array}{l}\text { LOD group } \\
(N=48)\end{array}$ & & \\
\hline \multicolumn{7}{|l|}{ Left } \\
\hline Medial orbitofrontal & 445 & 196.28 & $2.64(0.15)$ & $2.51(0.17)$ & -0.81 & $<0.00001$ \\
\hline Lateral occipital & 96 & 84.27 & $2.20(0.17)$ & $2.08(0.20)$ & -0.64 & $<0.0001$ \\
\hline Middle temporal & 85 & 58.16 & $3.10(0.18)$ & $2.96(0.21)$ & -0.72 & $<0.000$ । \\
\hline Paracentral & 112 & 50.31 & $2.36(0.17)$ & $2.24(0.20)$ & -0.64 & $<0.0001$ \\
\hline Supra marginal & 64 & 22.38 & $2.75(0.15)$ & $2.68(0.20)$ & -0.40 & $<0.00$ । \\
\hline Postcentral & 107 & 34.22 & $2.14(0.16)$ & $2.05(0.19)$ & -0.51 & $<0.001$ \\
\hline Rostral anterior cingulate & 83 & 42.72 & $2.58(0.16)$ & $2.49(0.16)$ & -0.56 & $<0.001$ \\
\hline Precuneus & 38 & 16.30 & $2.56(0.20)$ & $2.45(0.22)$ & -0.52 & $<0.001$ \\
\hline \multicolumn{7}{|l|}{ Right } \\
\hline Postcentral & 265 & 107.62 & $2.61(0.18)$ & $2.47(0.16)$ & -0.82 & $<0.000$ \\
\hline Pars opercularis & 264 & 154.40 & $2.73(0.14)$ & $2.59(0.20)$ & -0.81 & $<0.0001$ \\
\hline Rostral middle frontal & 42 & 29.12 & $2.66(0.14)$ & $2.57(0.15)$ & -0.62 & $<0.001$ \\
\hline Precuneus & 134 & 72.32 & $2.46(0.23)$ & $2.36(0.18)$ & -0.48 & $<0.001$ \\
\hline Isthmus cingulate & 71 & 35.15 & $2.67(0.21)$ & $2.53(0.27)$ & -0.57 & $<0.001$ \\
\hline Insula & 202 & 56.99 & $2.92(0.16)$ & $2.77(0.21)$ & -0.80 & $<0.0001$ \\
\hline
\end{tabular}

Abbreviations: LOD, late-onset depression; FDR, false discovery rate.

reduction in the drug-naive patients with LOD, relative to group-matched healthy controls. The strength of this study lies on the recruitment of relatively larger samples and drugnaive LOD patients compared with the other neuroimaging studies on LOD. Hence, we could investigate more on the subtle differences of cortical thickness/subcortical volumes between the LOD and controls, as well as the relationships between the cortical thickness/subcortical volumes and the various clinical/neuropsychological measurements, without interference of the medication effects. Indeed, there have been some suggestions that antidepressants may exert a neurotrophic effect on particular regions of the brain (Duman and Monteggia, 2006; Rocher et al, 2004; Stewart and Reid, 2000). Furthermore, in geriatric patients with depression, antidepressant exposure was associated with larger orbitofrontal gray matter volume as compared with medication-naive patients (Lavretsky et al, 2005).

\section{Cortical Thickness and Subcortical Volume Difference Between LOD and Control}

In this study, we have found the cortical thinning and subcortical volume reduction of the fronto-striatal-limbic structures in LOD patients as compared with healthy controls. These results were in line with the previous structural neuroimaging studies on depressive disorders, including LOD. The rostral anterior cingulate cortex is thought to be involved in assessing both emotional and motivational information and in the regulation of the emotional response (Bush et al, 2000). The orbitofrontal cortex dysfunction may yield the characteristic state of of depression, which shows impaired ability to interrupt perseverative melancholic thoughts and anxious responses to ordinarily nonthreatening stimuli (Ballmaier et al, 2004b; Ongr and Price, 2000). Deficit in the top-down inhibitory control of the DLPFC over the amygdala and sundry limbic tissue may result in chronic limbic overactivity and negative emotions (Davidson, 2002; Savitz and Drevets, 2009). Many studies reported smaller hippocampal volumes in patients with major depressive disorder as compared with healthy control subjects, which may stem from the dysfunctional hypothalamic-pituitary-adrenal (HPA) axis (MacQueen and Frodl, 2011; Vreeburg et al, 2009). The hippocampus plays an inhibitive role in regulating the HPA axis (Jacobson and Sapolsky, 1991), and chronic exposure to glucocorticoids with repeated depressive episodes could lead to cell death and hippocampal atrophy (Knoops et al, 2010; Sapolsky et al, 1986). 


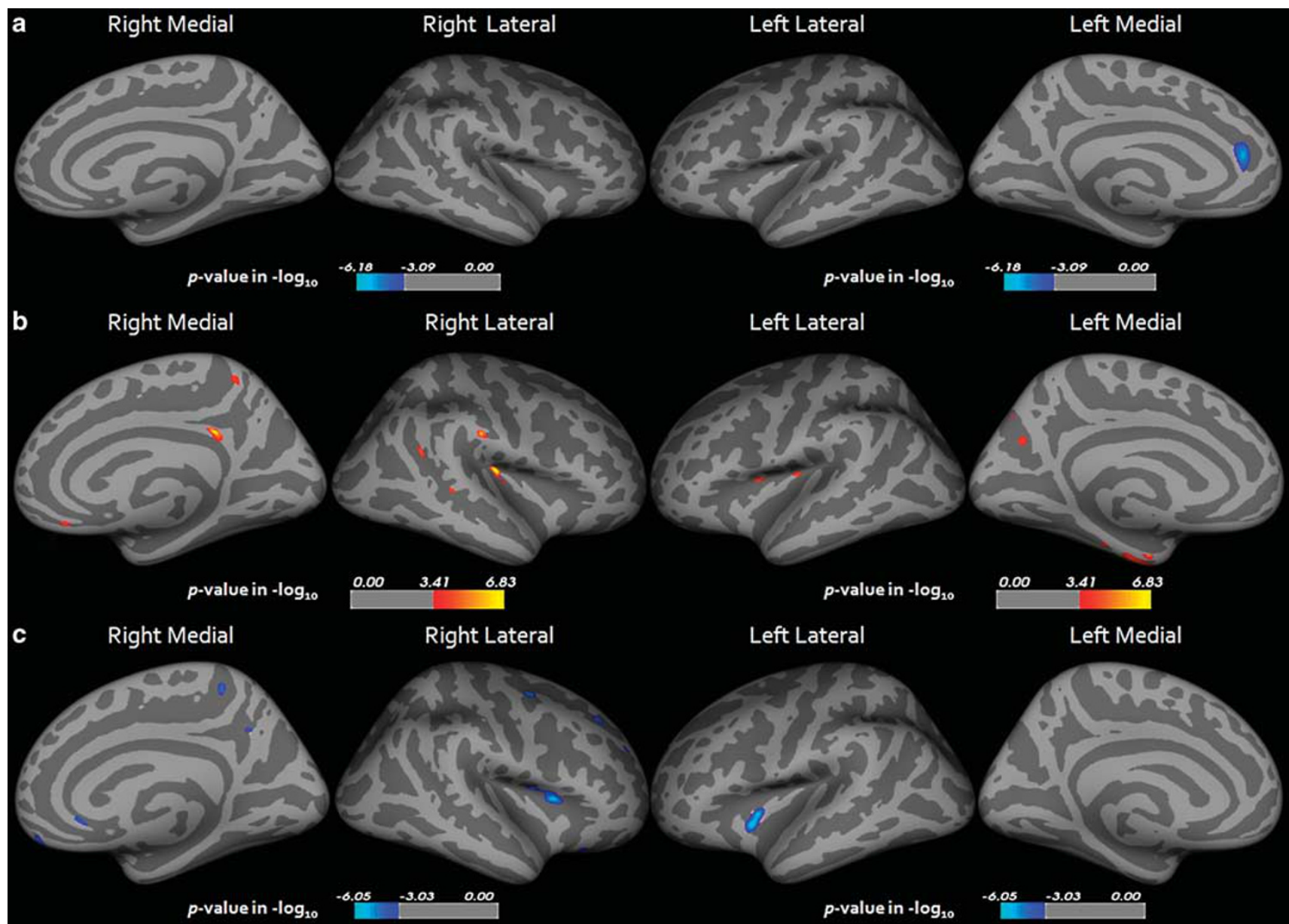

Figure 2 Statistical maps showing the regions of cortical thickness correlated with HAM-D 17 total score (a), CERAD-K word list recall score (b), and Stroop Word-Color Interference test score (c) in the LOD group ( $p<0.05$ FDR corrected). Maps are shown for right and left hemispheres in lateral and medial views respectively, and significant regions are shown in blue (negative correlation) and red (positive correlation). LOD, late-onset depression; CERAD-K, Korean version of Consortium to Establish a Registry for Alzheimer's Disease; HAM-D 17, Hamilton Depression Rating Scale; FDR, false discovery rate.

In addition to the fronto-striatal-limbic structural abnormalities, we also found the cortical thinning of the temporoparietal-limbic structures in LOD patients as compared with controls. Previous voxel-wise whole-brain structural neuroimaging studies have revealed reduced gray matter volumes in the temporo-parietal cortex in LOD patients, and suggested that these structural abnormalities might be the distinctive pattern of the LOD (Ballmaier et al, 2004a; Egger et al, 2008). More specifically, in this study, we found the reduced cortical thinning of the bilateral precuneus and the right posterior cingulate cortex in patients with LOD. The bulk of the previous structural neuroimaging studies have shown the precuneus and posterior cingulate structural and functional abnormalities in early $\mathrm{AD}$ and MCI (Fennema Notestine et al, 2009; McDonald et al, 2009; McEvoy et al, 2009; Mosconi, 2005). Moreover, the gray matter reductions in both the precuneus and the posterior cingulate cortex in patients with MCI were considered a predictor of the conversion to AD (Misra et al, 2009). Therefore, we suggest that the structural abnormalities found in these areas might explain the neurobiological mechanisms of LOD as a risk factor of dementia.

\section{Neurobiological Mechanisms of Brain Structural Changes in LOD}

To date, the glucocorticoid-induced fronto-striatal-limbic area dysfunction was considered the central neurobiological model of depression (Jacobson and Sapolsky, 1991; MacQueen and Frodl, 2011; Savitz and Drevets, 2009; Vreeburg et al, 2009). Additionally, cerebrovascular risk factors, such as hypertension, diabetes, and subcortical white matter hyperintensities, were known to play a crucial role in the physiopathology of LOD (Alexopoulos et al, 1988, 1997; Krishnan, 2002). In this study, we could minimize the significant effects of subcortical white matter hyperintensities in the LOD by exclusion of subjects with significant white matter hyperintensities and inclusion of subjects with normal-appearing white matter. In this regard, we might raise the possibilities of other putative mechanisms of the LOD, such as the $\beta$-amyloid- or tau proteininduced fronto-striatal-limbic disruption. A recent positron emission tomography study by Butters et al (2008a) demonstrated the Pittsburgh Compound B (PiB) retention in approximately one-half of 9 nondemented subjects with 
Table 4 Subcortical Volumes of Control Group and LOD Group

\begin{tabular}{|c|c|c|c|c|c|}
\hline \multirow{2}{*}{ Region } & \multicolumn{2}{|c|}{ Subcortical volume $\left(\mathrm{mm}^{3}, \mathrm{SD}\right)$} & \multirow{2}{*}{$\begin{array}{l}\text { Effect size } \\
\text { (Cohen's d) }\end{array}$} & \multirow{2}{*}{$\begin{array}{c}\text { Percent } \\
\text { difference }\end{array}$} & \multirow{2}{*}{$p$} \\
\hline & $\begin{array}{c}\text { Control group } \\
(N=47)\end{array}$ & $\begin{array}{l}\text { LOD group } \\
(N=48)\end{array}$ & & & \\
\hline \multicolumn{6}{|l|}{ Left } \\
\hline White matter & $219060.6(21135.9)$ & $215750.2(24$ 193.5) & -0.14 & $1.5 \%$ & NS \\
\hline Pallidum & $1616.8(202.6)$ & I $530.5(242.0)$ & -0.39 & $5.3 \%$ & NS \\
\hline Putamen & 4730.7 (537.9) & 4380.7 (792.5) & -0.52 & $7.4 \%$ & NS \\
\hline Hippocampus & $3738.2(393.1)$ & $3456.4(476.6)$ & -0.65 & $7.5 \%$ & NS \\
\hline Amygdala & | 489.2 (| 89.2) & | 327.5 (270.8) & -0.69 & $10.8 \%$ & NS \\
\hline Thalamus & $6146.4(544.5)$ & $5915.4(858.9)$ & -0.32 & $3.7 \%$ & NS \\
\hline Pallidum & | 462.2 (| 32.6) & | 385.6 (233.7) & -0.40 & $5.2 \%$ & NS \\
\hline Putamen & $4451.5(523.1)$ & $4185.0(584.6)$ & -0.48 & $5.9 \%$ & NS \\
\hline Hippocampus & $3995.4(380.9)$ & $3656.3(497.3)$ & -0.77 & $8.5 \%$ & 0.001 \\
\hline Amygdala & | 583.4 (238.9) & | 433.| (264.0) & -0.60 & $9.5 \%$ & NS \\
\hline
\end{tabular}

Abbreviations: LOD, late-onset depression; NS, statistically not significant.

Uncorrected $p<0.001$ was considered as significant.

treated depression and variable cognitive impairment, indicative of brain $\beta$-amyloid accumulation in cortical areas in a pattern characteristic of early AD. In addition, a postmortem study by Sweet et al, (2004) has confirmed the predominance of $\mathrm{AD}$ neuropathology among well-characterized LOD patients with varying cognitive impairment, who were followed longitudinally.

\section{Correlation Analyses with Clinical Outcomes and Neuropsychological Performance}

In this study, the depression severity in LOD measured by the $H A M-D_{17}$ total score was significantly correlated with the rostral anterior cingulate cortex, which was in line with the result from other study (Chen et al, 2007). In addition, a previous volumetric study on geriatric depression revealed that patients who failed to remit following escitalopram treatment had smaller dorsal and rostral anterior cingulate gray matter volumes than patients who remitted (Gunning et al, 2009). However, the neural substrates associated with the severity of depression have been rather inconsistent (Savitz and Drevets, 2009); this might be attributable to the difference in the subjects, depression scales, and chronic episodes. In our study, the Stroop Color-Word Interference test score, which was measuring executive function, of LOD patients showed significant inverse correlation with the rostral anterior cingulate, orbitofrontal cortex, and DLPFC. It has been demonstrated that the executive dysfunction is characteristic of the clinical presentation of most patients with LOD (Herrmann et al, 2007; Murphy and Alexopoulos, 2006), it remains present even after amelioration of depressive symptoms (Alexopoulos et al, 2005), and may predict the treatment response (Alexopoulos et al, 2005). The previous studies suggested that disruption in white matter integrity might be closely related to the executive dysfunction of LOD (Murphy and Alexopoulos, 2006). Although we showed the associations between the frontal cortical thinning and the executive functions, it is still unclear whether these associations are related to further clinical courses such as treatment response of LOD with the lack of white matter changes. Further longitudinal studies will be needed for clarification of these relationships. Through these, we will also be able to find the predictors of various clinical courses including treatment response.

In this study, we observed the VF deficits and significant positive correlations with the volumes of the putamen and the hippocampus in the LOD. A fundamental component of the CERAD VF task is the retrieval of semantically associated words from long-term memory storage (semantic fluency task). It has been recognized previously as one of the reflections of the frontal and the temporal lobe function (Baldo et al, 2006; Phelps et al, 1997). A previous study using single photon emission computed tomography (SPECT) showed the prefrontal and the hippocampal activations in depressed patients under the semantic fluency test conditions, compared with the phonemic fluency conditions (Audenaert et al, 2002). They suggested that depression patients might compensate for their dysfunctional prefrontal 'search' mechanisms by using more direct strategies to obtain entrance to their verbal hippocampal memory systems. In addition, significant correlations between the hippocampal volumes and semantic fluency task were observed in the patients with MCI and AD (Dos Santos et al, 2011; Gleissner and Elger, 2001). A previous 

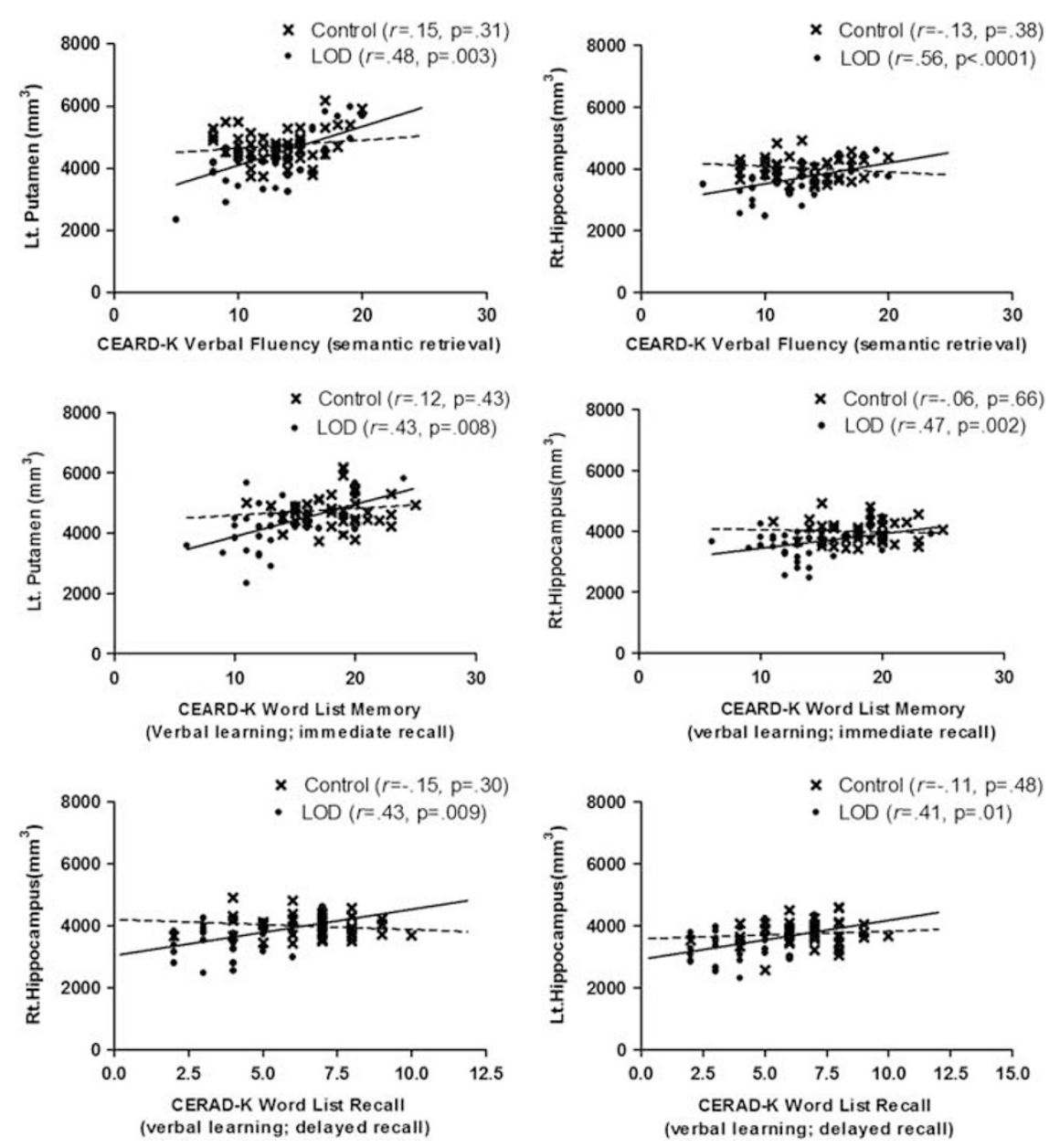

Figure 3 Relationships between CERAD-K VF, WLM, and WLR scores, and subcortical volumes. CERAD-K, Korean version of Consortium to Establish a Registry for Alzheimer's Disease; VF, Verbal Fluency; WLM, Word List Memory; WLR, Word List Recall.

voxel-based lesion symptom mapping study on the stroke patients showed that the scores of semantic fluency task were correlated with the putamen and the insula (Baldo et al, 2006). Our results indicate that both the hippocampus and the putamen were also engaged in the retrieval process during the semantic fluency task in LOD. To our best knowledge, there have been no previous structural neuroimaging studies on the semantic fluency task in depression. Therefore, it is not clear whether these relationships were distinct process in LOD or not. Further replication and longitudinal studies will be needed to clarify the relationships between the VF and the striatal-limbic structures in LOD.

\section{Clinical Implications of Cognitive Impairments in LOD}

In this study, several cognitive functions were significantly reduced in LOD patients compared with control group. Although the mean scores of several cognitive functions were within the normal range and did not reach the degree of MCI, these cognitive impairments, especially memory function impairment, might be meaningful in terms of the risk of dementia. The WLM (verbal learning; immediate recall) and WLR (verbal learning; delayed recall) scores were significantly correlated with the cortical thickness of the entorhinal cortex, the precuneus, and the isthmus cingulate in the LOD group but not in the control group, which were in line with the previous study on the MCI (Schmidt Wilcke et al, 2009). A hippocampal shape analysis by Ballmaier et al (2008) revealed that delayed recall scores were correlated significantly with the hippocampal volumes of LOD but not in early-onset elderly depression. In this study, we extended this previous result to the structural abnormalities of the other medial temporal and posterior parietal structures, which might be a distinctive feature of LOD as compared with early-onset elderly depression. A previous VBM study by Avila et al (2011) suggesting direct correlation between delayed visual-verbal memory recall scores with left parahippocampal volumes in elderly depressed individuals provided support to the view that depression in elderly populations may be a risk factor for dementia. As our results showed that depression severity was not correlated with the medial temporal and posterior parietal structures, these cognitive impairments might be an independent and co-occurring process in LOD (Butters et al, 2008b). However, previous researchers have also proposed that fronto-striatal dysfunction might exert a significant effect on cognitive impairments of LOD (Butters et al, 2008b). They suggested that the amyloid burden, vascular insults, and glucocorticoid insults to the frontostriatal circuits might be added to the total brain injury burden, lowering reserve and vulnerability to express 
cognitive impairments. Further longitudinal neuroimaging studies will be needed to prove these causal relationships of cognitive impairments and depressive symptoms. Taken together, these structural abnormalities of the medial temporal, the precuneus, and the posterior cingulate in LOD might explain the neurobiological mechanisms of LOD as a risk factor of dementia. Furthermore, longitudinal studies will be needed for investigation of the effectiveness of early intervention of LOD for the prevention of dementia.

\section{LIMITATIONS AND CONCLUSIONS}

The limitations of our study were as follows. First, the mean duration of LOD was relatively shorter in this study. Thus, we could not observe the relationship of cortical thinning/ subcortical volumes with disease duration. In this regard, the results of this study might be interpreted cautiously and should not be generalized to the chronic LOD patients; a longitudinal study is needed. Second, the number of female subjects was larger than male subjects in each group. Although the total brain volumes and cortical thickness were not significantly different between the male and female subjects, further investigation of the gender effect on the cortical thickness and subcortical volumes will be needed. Finally, although we suggested the possibility of LOD with cognitive impairment as a risk factor of dementia, we should bear in mind that cognitive impairments and structural changes in LOD could be temporary and improved after effective treatments such as antidepressants and cognitive training. Indeed, previous studies showed that antidepressants increased neurogenesis in adult hippocampal neurons (Anacker et al, 2011; Boldrini et al, 2009). Although no data are available for elderly depression, antidepressant treatment is known to increase hippocampal volume in posttraumatic stress disorder (Bossini et al, 2007). In addition, there were also evidences suggesting increase in cortical thickness of the healthy elderly by cognitive training (Engvig et al, 2010). Therefore, further longitudinal structural neuroimaging studies, thorough observations of clinical courses, and precise patient stratification throughout the molecular imaging (ie, PiB), and analysis of CSF $\beta$-amyloid and tau protein will be needed for clarification of our hypothesis.

In conclusion, we showed cortical thickness and subcortical volume reductions in multiple fronto-striatal-limbic structures and various temporo-parietal-limbic structures in LOD. In addition, we also showed significant correlations between the cortical thickness of the medial temporal, the posterior cingulate, and the precuneus and the episodic memory functions in LOD. These structural changes might explain the neurobiological mechanisms of LOD as a risk factor of dementia. Cortical thinning observed in this study may be related to impairment of emotional and cognitive processing in LOD, but longitudinal studies will be necessary to confirm this hypothesis.

\section{ACKNOWLEDGEMENTS}

This work was supported by a grant from the NextGeneration BioGreen 21 Program (No. PJ007186), Rural Development Administration, Republic of Korea.

\section{DISCLOSURE}

The authors declare no conflict of interest.

\section{REFERENCES}

Alberti KG, Zimmet P, Shaw J (2005). The metabolic syndrome a new worldwide definition. Lancet 366: 1059-1062.

Alexopoulos GS, Kiosses DN, Heo M, Murphy CF, Shanmugham B, Gunning Dixon F (2005). Executive dysfunction and the course of geriatric depression. Biol Psychiatry 58: 204-210.

Alexopoulos GS, Meyers BS, Young RC, Campbell S, Silbersweig D, Charlson M (1997). 'Vascular depression' hypothesis. Arch Gen Psychiatry 54: 915-922.

Alexopoulos GS, Young RC, Meyers BS, Abrams RC, Shamoian CA (1988). Late-onset depression. Psychiatr Clin North Am 11: 101-115.

Anacker C, Zunszain PA, Cattaneo A, Carvalho LA, Garabedian MJ, Thuret $S$ et al (2011). Antidepressants increase human hippocampal neurogenesis by activating the glucocorticoid receptor. Mol Psychiatry 16: 738-750.

Apostolova LG, Cummings JL (2008). Neuropsychiatric manifestations in mild cognitive impairment: a systematic review of the literature. Dement Geriatr Cogn Disord 25: 115-126.

Ashburner J, Csernansky JG, Davatzikos C, Fox NC, Frisoni GB, Thompson PM (2003). Computer-assisted imaging to assess brain structure in healthy and diseased brains. Lancet Neurol 2: 79-88.

Audenaert K, Goethals I, Van Laere K, Lahorte P, Brans B, Versijpt $J$ et al (2002). SPECT neuropsychological activation procedure with the Verbal Fluency Test in attempted suicide patients. Nucl Med Commun 23: 907-916.

Avila R, Ribeiz S, Duran FL, Arrais JP, Moscoso MA, Bezerra DM et al (2011). Effect of temporal lobe structure volume on memory in elderly depressed patients. Neurobiol Aging 32: 1857-1867.

Baldo JV, Schwartz S, Wilkins D, Dronkers NF (2006). Role of frontal versus temporal cortex in verbal fluency as revealed by voxel-based lesion symptom mapping. J Int Neuropsychol Soc 12: 896-900.

Ballmaier M, Kumar A, Thompson PM, Narr KL, Lavretsky H, Estanol L et al (2004a). Localizing gray matter deficits in lateonset depression using computational cortical pattern matching methods. Am J Psychiatry 161: 2091-2099.

Ballmaier M, Narr KL, Toga AW, Elderkin Thompson V, Thompson PM, Hamilton L et al (2008). Hippocampal morphology and distinguishing late-onset from early-onset elderly depression. Am J Psychiatry 165: 229-237.

Ballmaier M, Toga AW, Blanton RE, Sowell ER, Lavretsky H, Peterson J et al (2004b). Anterior cingulate, gyrus rectus, and orbitofrontal abnormalities in elderly depressed patients: an MRI-based parcellation of the prefrontal cortex. Am J Psychiatry 161: 99-108.

Boldrini M, Underwood MD, Hen R, Rosoklija GB, Dwork AJ, John Mann J et al (2009). Antidepressants increase neural progenitor cells in the human hippocampus. Neuropsychopharmacology 34: 2376-2389.

Bossini L, Tavanti M, Lombardelli A, Calossi S, Polizzotto NR, Galli $\mathrm{R}$ et al (2007). Changes in hippocampal volume in patients with post-traumatic stress disorder after sertraline treatment. J Clin Psychopharmacol 27: 233-235.

Brown RP, Sweeney J, Loutsch E, Kocsis J, Frances A (1984). Involutional melancholia revisited. Am J Psychiatry 141: 24-28.

Bush G, Luu P, Posner MI (2000). Cognitive and emotional influences in anterior cingulate cortex. Trends Cogn Sci 4: 215-222.

Butters MA, Klunk WE, Mathis CA, Price JC, Ziolko SK, Hoge JA et al (2008a). Imaging Alzheimer pathology in late-life depression with PET and Pittsburgh Compound-B. Alzheimer Dis Assoc Disord 22: 261-268. 
Butters MA, Young JB, Lopez O, Aizenstein HJ, Mulsant BH, Reynolds CF et al (2008b). Pathways linking late-life depression to persistent cognitive impairment and dementia. Dialogues Clin Neurosci 10: 345-357.

Chen C, Ridler K, Suckling J, Williams S, Fu CH, Merlo Pich E et al (2007). Brain imaging correlates of depressive symptom severity and predictors of symptom improvement after antidepressant treatment. Biol Psychiatry 62: 407-414.

Chobanian AV, Bakris GL, Black HR, Cushman WC, Green LA, Izzo JL et al (2003). The seventh report of the joint national committee on prevention, detection, evaluation, and treatment of high blood pressure: the JNC 7 report. JAMA (Chicago III) 289: 2560-2572.

Crocco EA, Castro K, Loewenstein DA (2010). How late-life depression affects cognition: neural mechanisms. Curr Psychiatry Rep 12: 34-38.

Dale AM, Fischl B, Sereno MI (1999). Cortical surface-based analysis. I. Segmentation and surface reconstruction. NeuroImage 9: 179-194.

Davidson RJ (2002). Anxiety and affective style: role of prefrontal cortex and amygdala. Biol Psychiatry 51: 68-80.

Desikan RS, Sgonne F, Fischl B, Quinn BT, Dickerson BC, Blacker $\mathrm{D}$ et al (2006). An automated labeling system for subdividing the human cerebral cortex on MRI scans into gyral based regions of interest. NeuroImage 31: 968-980.

Dos Santos V, Thomann PA, Wstenberg T, Seidl U, Essig M, Schrder J (2011). Morphological cerebral correlates of CERAD test performance in mild cognitive impairment and Alzheimer's disease. J Alzheimers Dis 23: 411-420.

Duman RS, Monteggia LM (2006). A neurotrophic model for stress-related mood disorders. Biol Psychiatry 59: 1116-1127.

Egger K, Schocke M, Weiss E, Auffinger S, Esterhammer R, Goebel $\mathrm{G}$ et al (2008). Pattern of brain atrophy in elderly patients with depression revealed by voxel-based morphometry. Psychiatry Res 164: 237-244.

Emery VO, Oxman TE (1992). Update on the dementia spectrum of depression. Am J Psychiatry 149: 305-317.

Engvig A, Fjell AM, Westlye LT, Moberget T, Sundseth VA, Larsen $\mathrm{KB}$ et al (2010). Effects of memory training on cortical thickness in the elderly. NeuroImage 52: 1667-1676.

Fennema Notestine C, Hagler DJ, McEvoy LK, Fleisher AS, Wu EH, Karow DS et al (2009). Structural MRI biomarkers for preclinical and mild Alzheimer's disease. Hum Brain Mapp 30: 3238-3253.

Fischl B, Dale AM (2000). Measuring the thickness of the human cerebral cortex from magnetic resonance images. Proc Natl Acad Sci USA 97: 11050-11055.

Fischl B, Sereno MI, Tootell RB, Dale AM (1999). High-resolution intersubject averaging and a coordinate system for the cortical surface. Hum Brain Mapp 8: 272-284.

Fischl B, van der Kouwe A, Destrieux C, Halgren E, Sgonne F, Salat DH et al (2004). Automatically parcellating the human cerebral cortex. Cereb Cortex 14: 11-22.

Genovese CR, Lazar NA, Nichols T (2002). Thresholding of statistical maps in functional neuroimaging using the false discovery rate. NeuroImage 15: 870-878.

Gleissner U, Elger CE (2001). The hippocampal contribution to verbal fluency in patients with temporal lobe epilepsy. Cortex 37: 55-63.

Grundy SM, Cleeman JI, Daniels SR, Donato KA, Eckel RH, Franklin BA et al (2005). Diagnosis and management of the metabolic syndrome: an American Heart Association/National Heart, Lung, and Blood Institute Scientific Statement. Circulation 112: 2735-2752.

Gunning FM, Cheng J, Murphy CF, Kanellopoulos D, Acuna J, Hoptman MJ et al (2009). Anterior cingulate cortical volumes and treatment remission of geriatric depression. Int J Geriatr Psychiatry 24: 829-836.

Hamilton M (1967). Development of a rating scale for primary depressive illness. Br J Soc Clin Psychol 6: 278-296.
Han X, Jovicich J, Salat D, van der Kouwe A, Quinn B, Czanner S et al (2006). Reliability of MRI-derived measurements of human cerebral cortical thickness: the effects of field strength, scanner upgrade and manufacturer. NeuroImage 32: 180-194.

Herrmann L, Goodwin GM, Ebmeier KP (2007). The cognitive neuropsychology of depression in the elderly. Psychol Med 37: 1693-1702.

Huang J, Friedland RP, Auchus AP (2007). Diffusion tensor imaging of normal-appearing white matter in mild cognitive impairment and early Alzheimer disease: preliminary evidence of axonal degeneration in the temporal lobe. AJNR Am J Neuroradiol 28: 1943-1948.

Hutton C, Draganski B, Ashburner J, Weiskopf N (2009). A comparison between voxel-based cortical thickness and voxelbased morphometry in normal aging. NeuroImage 48: 371-380.

Hwang J, Lee T, Tsai S, Chen T, Yang C, Lirng J et al (2010). Cortical and subcortical abnormalities in late-onset depression with history of suicide attempts investigated with MRI and voxelbased morphometry. J Geriatr Psychiatry Neurol 23: 171-184.

Jacobson L, Sapolsky R (1991). The role of the hippocampus in feedback regulation of the hypothalamic-pituitary-adrenocortical axis. Endocr Rev 12: 118-134.

Janssen J, Hulshoff Pol HE, de Leeuw F, Schnack HG, Lampe IK, Kok RM et al (2007). Hippocampal volume and subcortical white matter lesions in late life depression: comparison of early and late onset depression. J Neurol Neurosurg Psychiatry 78: 638-640.

Jovicich J, Czanner S, Han X, Salat D, van der Kouwe A, Quinn B et al (2009). MRI-derived measurements of human subcortical, ventricular and intracranial brain volumes: reliability effects of scan sessions, acquisition sequences, data analyses, scanner upgrade, scanner vendors and field strengths. NeuroImage 46: 177-192.

Knoops AJ, Gerritsen L, van der Graaf Y, Mali WP, Geerlings MI (2010). Basal hypothalamic pituitary adrenal axis activity and hippocampal volumes: the SMART-Medea study. Biol Psychiatry 67: 1191-1198.

Koolschijn PC, van Haren NE, Lensvelt-Mulders GJ, Hulshoff Pol HE, Kahn RS (2009). Brain volume abnormalities in major depressive disorder: a meta-analysis of magnetic resonance imaging studies. Hum Brain Mapp 30: 3719-3735.

Koolschijn PC, van Haren NE, Schnack HG, Janssen J, Hulshoff Pol HE, Kahn RS (2010). Cortical thickness and voxel-based morphometry in depressed elderly. Eur Neuropsychopharmacol 20: 398-404.

Krishnan KR (2002). Biological risk factors in late life depression. Biol Psychiatry 52: 185-192.

Krishnan KR, Hays JC, Blazer DG (1997). MRI-defined vascular depression. Am J Psychiatry 154: 497-501.

Krishnan KR, Hays JC, Tupler LA, George LK, Blazer DG (1995). Clinical and phenomenological comparisons of late-onset and early-onset depression. Am J Psychiatry 152: 785-788.

Lavretsky H, Roybal DJ, Ballmaier M, Toga AW, Kumar A (2005). Antidepressant exposure may protect against decrement in frontal gray matter volumes in geriatric depression. J Clin Psychiatry 66: 964-967.

Lee JH, Lee KU, Lee DY, Kim KW, Jhoo JH, Kim JH et al (2002). Development of the Korean version of the Consortium to Establish a Registry for Alzheimer's Disease Assessment Packet (CERAD-K): clinical and neuropsychological assessment batteries. J Gerontol B Psychol Sci Soc Sci 57: P47-P53.

Lerch JP, Pruessner JC, Zijdenbos A, Hampel H, Teipel SJ, Evans AC (2005). Focal decline of cortical thickness in Alzheimer's disease identified by computational neuroanatomy. Cereb Cortex 15: 995-1001.

Lyoo IK, Sung YH, Dager SR, Friedman SD, Lee J, Kim SJ et al (2006). Regional cerebral cortical thinning in bipolar disorder. Bipolar Disord 8: 65-74.

MacQueen G, Frodl T (2011). The hippocampus in major depression: evidence for the convergence of the bench and bedside in psychiatric research? Mol Psychiatry 16: 252-264. 
McDonald CR, McEvoy LK, Gharapetian L, Fennema Notestine C, Hagler DJ, Holland D et al (2009). Regional rates of neocortical atrophy from normal aging to early Alzheimer disease. Neurology 73: 457-465.

McEvoy LK, Fennema Notestine C, Roddey JC, Hagler DJ, Holland D, Karow DS et al (2009). Alzheimer disease: quantitative structural neuroimaging for detection and prediction of clinical and structural changes in mild cognitive impairment. Radiology 251: 195-205.

Misra C, Fan Y, Davatzikos C (2009). Baseline and longitudinal patterns of brain atrophy in MCI patients, and their use in prediction of short-term conversion to $\mathrm{AD}$ : results from ADNI. NeuroImage 44: 1415-1422.

Modrego PJ, Ferrndez J (2004). Depression in patients with mild cognitive impairment increases the risk of developing dementia of Alzheimer type: a prospective cohort study. Arch Neurol 61: 1290-1293.

Morris JC (1993). The Clinical Dementia Rating (CDR): current version and scoring rules. Neurology 43: 2412-2414.

Mosconi L (2005). Brain glucose metabolism in the early and specific diagnosis of Alzheimer's disease. FDG-PET studies in MCI and AD. Eur J Nucl Med Mol Imaging 32: 486-510.

Murphy CF, Alexopoulos GS (2006). Attention network dysfunction and treatment response of geriatric depression. J Clin Exp Neuropsychol 28: 96-100.

Ongr D, Price JL (2000). The organization of networks within the orbital and medial prefrontal cortex of rats, monkeys and humans. Cereb Cortex 10: 206-219.

Park J, Kwon Y (1990). Modification of the mini-mental state examination for use in the elderly in a non-western society: Part I. Development of Korean version of Mini-Mental State Examination. Int J Geriatr Psychiatry 5: 381-387.

Phelps EA, Hyder F, Blamire AM, Shulman RG (1997). FMRI of the prefrontal cortex during overt verbal fluency. NeuroReport 8: 561-565.

Ries ML, Wichmann A, Bendlin B, Johnson SC (2009). Posterior cingulate and lateral parietal gray matter volume in older adults with depressive symptoms. Brain Imaging Behav 3: 233-239.
Rocher C, Spedding M, Munoz C, Jay TM (2004). Acute stressinduced changes in hippocampal/prefrontal circuits in rats: effects of antidepressants. Cereb Cortex 14: 224-229.

Salloway S, Malloy P, Kohn R, Gillard E, Duffy J, Rogg J et al (1996). MRI and neuropsychological differences in early- and late-life-onset geriatric depression. Neurology 46: 1567-1574.

Sapolsky RM, Krey LC, McEwen BS (1986). The neuroendocrinology of stress and aging: the glucocorticoid cascade hypothesis. Endocr Rev 7: 284-301.

Savitz J, Drevets WC (2009). Bipolar and major depressive disorder: neuroimaging the developmental-degenerative divide. Neurosci Biobehav Rev 33: 699-771.

Schmidt Wilcke T, Poljansky S, Hierlmeier S, Hausner J, Ibach B (2009). Memory performance correlates with gray matter density in the ento-/perirhinal cortex and posterior hippocampus in patients with mild cognitive impairment and healthy controls-a voxel based morphometry study. NeuroImage 47: 1914-1920.

Schweitzer I, Tuckwell V, O'Brien J, Ames D (2002). Is late onset depression a prodrome to dementia? Int J Geriatr Psychiatry 17: 997-1005.

Sheehan DV, Lecrubier Y, Sheehan KH, Amorim P, Janavs J, Weiller E et al (1998). The Mini-International Neuropsychiatric Interview (M.I.N.I.): the development and validation of a structured diagnostic psychiatric interview for DSM-IV and ICD-10. J Clin Psychiatry 59(Suppl 20): 22-33; quiz 34-57.

Sheline YI (2003). Neuroimaging studies of mood disorder effects on the brain. Biol Psychiatry 54: 338-352.

Stewart CA, Reid IC (2000). Repeated ECS and fluoxetine administration have equivalent effects on hippocampal synaptic plasticity. Psychopharmacology 148: 217-223.

Stroop JR (1935). Studies of interference in serial verbal reactions. J Exp Psychol 18: 643-662.

Sweet RA, Hamilton RL, Butters MA, Mulsant BH, Pollock BG, Lewis DA et al (2004). Neuropathologic correlates of late-onset major depression. Neuropsychopharmacology 29: 2242-2250.

Vreeburg SA, Hoogendijk WJ, van Pelt J, Derijk RH, Verhagen JC, van Dyck R et al (2009). Major depressive disorder and hypothalamic-pituitary-adrenal axis activity: results from a large cohort study. Arch Gen Psychiatry 66: 617-626.

Supplementary Information accompanies the paper on the Neuropsychopharmacology website (http://www.nature.com/npp) 